\title{
Influence of diffractive interactions on cosmic ray air showers
}

\author{
R. Luna* and A. Zepeda \\ Departamento de Física, Cinvestav, Av. IPN 2508, \\ Col. San Pedro Zacatenco, 07360 México DF, México. \\ C. A. García Canal and S. J. Sciutto \\ Departamento de Física and IFLP/CONICET, \\ Universidad Nacional de La Plata, C. C. 67 - 1900 La Plata, Argentina.
}

\begin{abstract}
A comparative study of commonly used hadronic collision simulation packages is presented. The characteristics of the products of hadron-nucleus collisions are analyzed from a general perspective, but focusing on their correlation with diffractive processes. One of the purposes of our work is to give quantitative estimations of the impact that different characteristics of the hadronic models have on air shower observables. Several sets of shower simulations using different settings for the parameters controlling the diffractive processes are used to analyze the correlations between diffractivity and shower observables. We find that the relative probability of diffractive processes during the shower development have a non negligible influence over the longitudinal profile as well as the distribution of muons at ground level. The implications on experimental data analysis are discussed.
\end{abstract}

PACS numbers: 13.85.-t, 13.85.Tp, 96.40.Pq, 07.05.Tp

\footnotetext{
* Also at ESCOM-IPN, México DF, México.
} 


\section{INTRODUCTION}

The measurement of extensive air showers (EAS) is presently the way to study cosmic rays with primary energy above several hundreds of $\mathrm{TeV}$. The properties of primary cosmic rays have to be deduced from the development of the shower in the atmosphere, and from the characteristics of the secondaries detected at the observation level.

Due to the lack of experimental data on particle interactions at the highest energies, it is necessary to interpret EAS measurements by comparing them with model predictions. Due to the complexity of the interactions that take place during the shower development, such predictions are generally obtained from Monte Carlo simulations.

The algorithms used to perform such simulations include sections that correspond to the different interactions that the secondary particles can undergo during their propagation. Among them, the hadronic interactions are one of the most difficult to model accurately, while playing a key role when trying to predict the final observables of a shower [1]. Such models are affected by uncertainties that cannot, at present, be totally controlled [2]. The uncertainties come from approximations that are intrinsic to the respective models, plus uncertainties and inconsistencies in the experimental data used to calibrate model parameters, plus the uncertainties associated with extrapolations outside the range covered by the available experimental data.

An important task is, therefore, to give quantitative estimation of the impact of these uncertainties on shower observables and on the estimations of properties of the primary particle. This question is of particular importance in ultra high energy cosmic ray observatories such as the Pierre Auger and even more relevant for primary energy determination from fluorescence emitance since this effect depends on the fraction of the cascade that produces the greatest portion of fluorescence light and in turn this fraction depends on the hadronic model.

The aim of the present work is aligned in this direction: well-known packages like SIBYLL [3-5], QGSJET [6, 7], and DPMJET [8-11] are extensively compared. Our study is carried

out with a very practical approach, analyzing first the secondaries produced after individual collisions, and then measuring the impact in the shower development of different hadronic configurations. This work covers several aspects of the hadronic interactions, but it is particularly concentrated in the study of the diffractive interactions. The analysis here 
presented is complementary to a work reported in references $[1,12]$.

In section II hadronic collisions, and in particular diffractive ones, are described. In section III we compare the properties of DPMJET, SYBILL and QGSJET hadronic models in collisions of protons with air with energy from $10^{2}$ to $10^{9} \mathrm{GeV}$. In section IV we compare the impact that the different alternatives for modeling the diffractive hadronic interactions have on common air shower observables. In section $\mathrm{V}$ we present our final remarks and conclusions.

\section{HADRONIC COLLISIONS}

From a practical point of view, a hadronic collision can be described as a process where an incident particle $P$, called the projectile, interacts with a target $A$-normally a nucleus of $A=Z+N$ nucleons ( $Z$ protons and $N$ neutrons)- to produce $N_{\text {sec }}$ secondary particles $S_{1}, \ldots, S_{N_{\mathrm{sec}}}$. Such secondary particles are generally hadrons, but can eventually be nuclear fragments, photons, etc., depending on the characteristics of the collision. In those cases where the primary particle survives after the collision (with changed energy and momentum), the surviving primary is accounted just as one more secondary particle within the $S_{1}, \ldots, S_{N_{\mathrm{sec}}}$ set.

Each secondary $S_{i}\left(i=1, \ldots, N_{\mathrm{sec}}\right)$ is characterized by its particle type, its energy $E_{S_{i}}$, and the angle $\theta_{i}$ between the primary and secondary momenta. It is also possible to define an azimuthal angle for each secondary. To adequately quantify the directions of the secondary particles it is convenient to use the so-called pseudorapidity, defined as $\eta=-\ln \tan (\theta / 2)$, instead of specifying the angle $\theta$ directly.

Let $E_{\text {lead }}$ be the energy of the secondary with maximum energy (the so-called leading particle). The leading energy fraction, $f_{L}$, for the collision, is defined as

$$
f_{L}=\frac{E_{\text {lead }}}{E_{P}}
$$

For high energy primaries the energies of the secondaries satisfy $\sum_{i=1}^{N_{\text {sec }}} E_{S_{i}} \leq E_{P}$ and, as a consequence, one has $0<f_{L}<1$. The inelasticity, $k_{\text {inel }}$, is defined as the fraction of energy carried by all the secondary particles, excluding the leading secondary,

$$
k_{\text {inel }}=1-f_{L} \text {. }
$$


In normal hadronic collisions $f_{L}$ or, equivalently, the inelasticity, fluctuates virtually in all the allowed range from 0 to 1 . In one extreme one has the hard interactions with large momentum transfers producing many secondaries that share the available primary energy. On the other hand, the so-called diffractive dissociation events, are characterized by low multiplicity and fast secondary particles, that imply $f_{L}$ close to 1 .

The diffractive interactions play a very important role during the development of air showers, due to the fact that they provide a way of transporting substantial amounts of energy deep in the atmosphere, and turn into a critical factor that controls the global characteristics of the shower profile [1].

The results coming from different theoretical treatments of soft interactions are not always coincident; and they cannot be conclusively checked against experimental data because up to the present time these forward processes could not be measured with enough accuracy in collider experiments $[2,12]$.

For all these reasons we consider important to investigate the properties of different quantities associated with hadronic interactions or air shower development, taking into account explicitly the diffractive or non-diffractive nature of the corresponding hadronic processes.

As mentioned before, in a diffractive collision there is a leading particle whose energy is clearly larger than the energies of the other secondaries ( $f_{L}$ close to 1$)$. Additionally, the total number of secondaries is generally small. On the other hand, a properly inelastic collision at very high energies is characterized by a large number of secondaries of comparable energy $\left(f_{L} \ll 1\right)$. We can therefore make simultaneous use of $N_{\text {sec }}$ and $f_{L}$, or equivalently $k_{\text {inel }}$, to distinguish diffractive from inelastic collisions. This approach proves to work acceptably in practice, having the advantage of being applicable to every collision generator (and even to the analysis of experimental data) where usually there is no additional information that permits discriminating the cases of true diffractive processes from the other non-diffractive ones.

\section{COMPARATIVE ANALYSIS OF HADRONIC MODELS}

As a first step in our study, we have performed a comparative analysis of the output coming from different hadronic packages when running them with a common input.

We have run batches of $N_{\text {coll }}$ events ( $N_{\text {coll }}=10,000$ unless otherwise specified) for each 

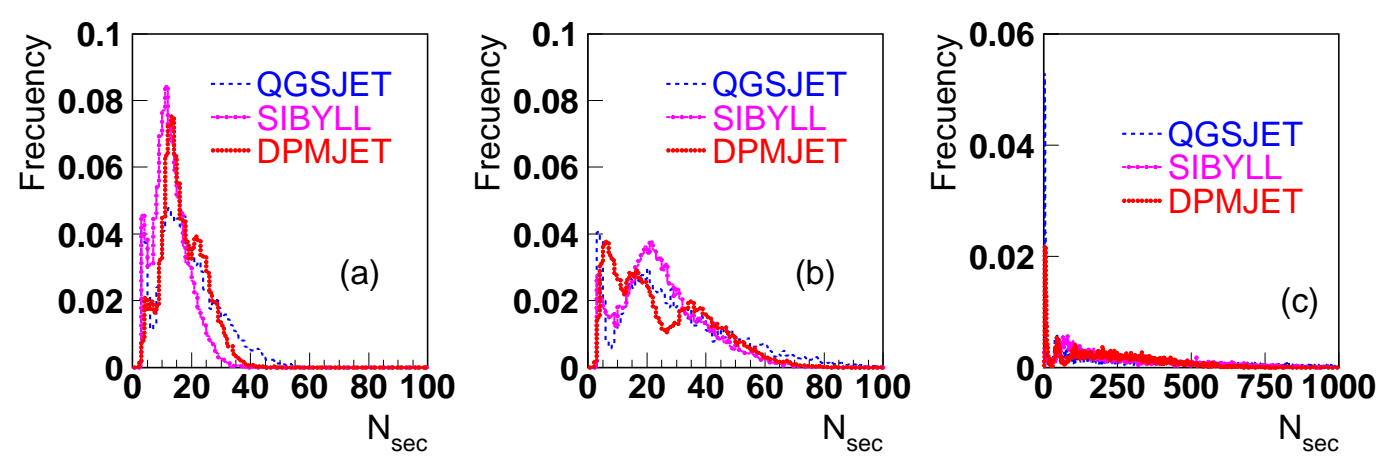

Figure 1: $N_{\text {sec }}$ distributions for proton-air collisions at $100 \mathrm{GeV}$ (a), $1 \mathrm{TeV}$ (b), and $100 \mathrm{PeV}$ (c).

combination of primary type, primary energy, and hadronic package. After each call to the hadronic procedures, a list of secondaries was obtained, with short-lived products (resonances) forced to decay. These secondaries were then processed to identify the leading particle, and to plot in histograms the quantities introduced in section II. Finally $f_{L}$ was evaluated, and analyzed in combination with the number of secondaries and the properties of the leading particle in order to label the collisions as "diffractive" or "non-diffractive". We have included in our analysis three of the most popular high energy interaction models, namely SIBYLL 2.1 [3], QGSJET01[6], and DPMJET 2.5 [8].

Every one of these models is capable of processing hadronic collisions having a hadron as projectile, and a specified nucleus as target. The energy of the particle must lie in a determined range, characteristic of each model. In the present work these ranges have been taken as $E_{P} \geq 30 \mathrm{GeV}$ for DPMJET and QGSJET, and $E_{P} \geq 100 \mathrm{GeV}$ for SIBYLL. With respect to the targets, we use a mixture of nitrogen and oxygen to emulate collisions in air, the medium of propagation of cosmic ray air showers.

\section{A. Multiplicity and inelasticity}

To start with our analysis of individual collisions, let us consider first the number of secondaries.

In figure 1, distributions of numbers of secondaries are displayed for several representative primary energies. The diffractive interactions show up clearly at each plot as a characteristic peak in the few-secondary zone of the abscissas. We can see that there are evident differences among the plots corresponding to different models, especially when comparing QGSJET with 


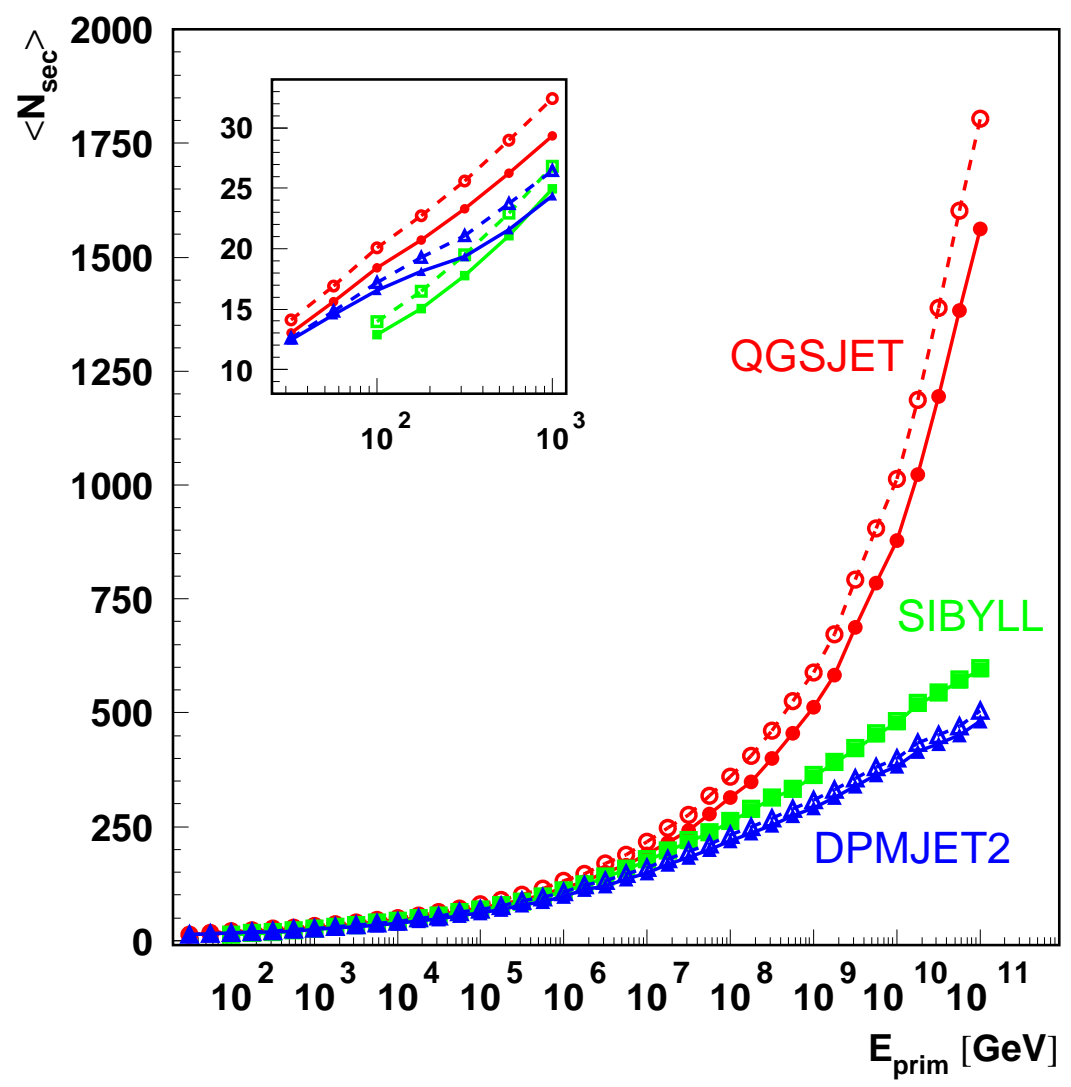

Figure 2: Average number of secondaries in proton-air collisions versus primary energy. The solid (open) symbols correspond to averages over all (non-diffractive) events. The lines are only to guide the eye. The low energy region is shown in more detail in the inset.

the other models. An outstanding feature is the well known fact that QGSJET produces substantially more secondaries than SIBYLL or DPMJET, especially at very high energies $[12,14]$. This fact shows up clearly in figure 2 where the average number of secondaries is plotted versus the primary energy. The curves with solid lines and symbols correspond to averages considering all kinds of events, while the curves with dashed lines and open symbols correspond to averages over non-diffractive events only.

The general averages are always smaller than the ones over non-diffractive events, as expected, since diffractive events have very few secondaries and therefore tend to reduce averages when included in the samples.

The differences between general and non-diffractive cases are significant in the case of QGSJET, small in the case of DPMJET, and almost negligible in the case of SIBYLL. A 


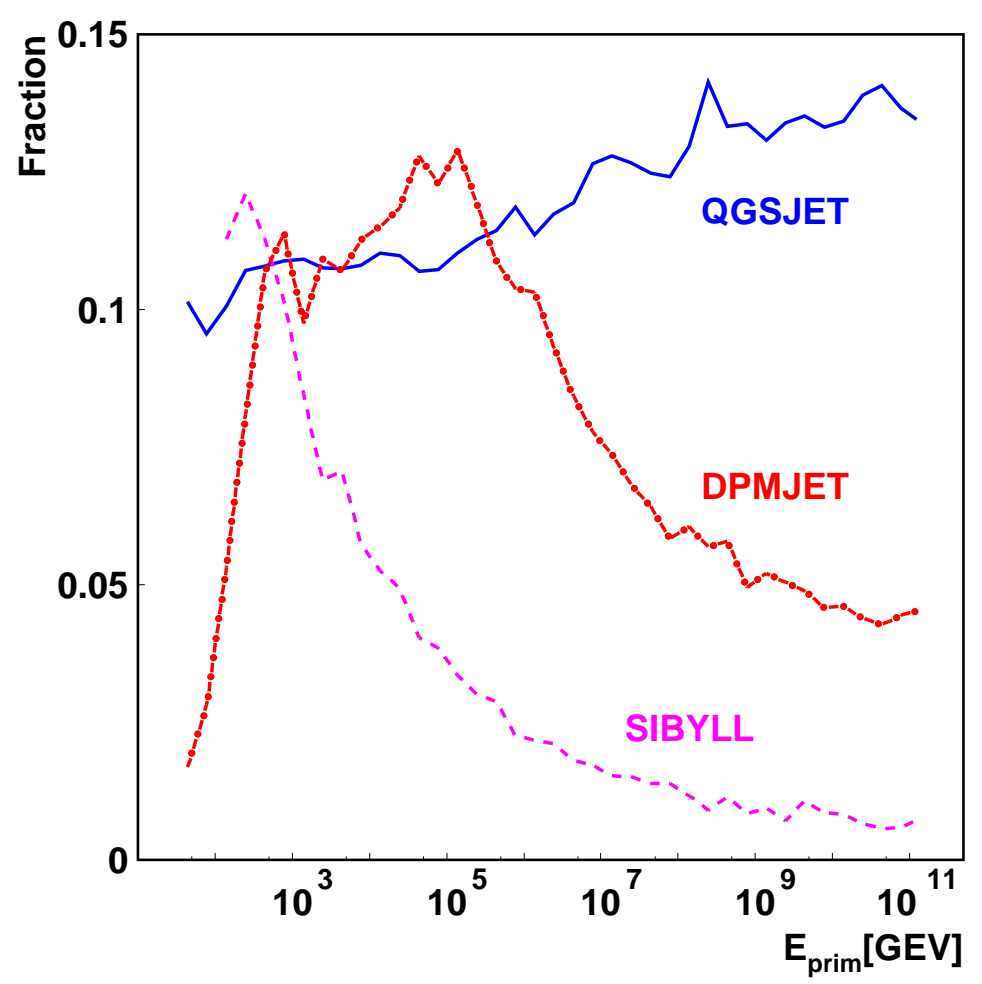

Figure 3: Fraction of diffractive events versus primary energies for the case of proton-air collisions.

similar behaviour can be found in the case of pion primaries (plots not included for brevity).

The main characteristics of the preceding plot are better understood considering that the influence of diffractive events in a sample is not only due to the properties of the diffractive interaction itself, but also to the magnitude of their relative probability. In figure 3, the fractions of diffractive events registered in our runs is plotted as a function of primary energy, in the case of proton primaries. The very significant difference between the QGSJET and SIBYLL cases is one of the outstanding features of this plot: these results indicate that in QGSJET the ratio between the diffractive and total cross sections does not suffer substantial variations in the whole range of energies considered (from $30 \mathrm{GeV}$ to $100 \mathrm{EeV}$ ), while the corresponding cross section ratio for SIBYLL presents a completely different behavior, decreasing as the primary energy increases. This explains clearly why the SIBYLL and DPMJET curves in figure 2 virtually overlap at the highest energies. Notice also that the relatively high diffractive probability of QGSJET tends to compensate the very large number of secondaries produced by this model in non diffractive interactions.

In between of these two completely different behaviors we can place the DPMJET case, 
characterized by a diffractive probability similar to QGSJET, for primary energies up to $10^{15} \mathrm{eV}$ approximately, and then decreasing continuously for larger primary energies.

It is important to mention that in the case of pion collisions, the probabilities of diffractive interactions in the cases considered (not plotted here for brevity) are very similar to the respective ones for protons.

The SIBYLL and QGSJET curves in figure 3 can be understood analysing the energy dependence of the diffractive, $\sigma_{\text {diff }}$, and total, $\sigma_{\text {tot }}=\sigma_{\text {diff }}+\sigma_{\text {inel }}$, cross sections, and taking into account that the fractions of diffractive events, $F_{\text {diff }}$, plotted at the mentioned figure are approximately equal to the respective diffractive to total cross section ratios, that is,

$$
F_{\text {diff }} \approx \frac{\sigma_{\text {diff }}}{\sigma_{\text {tot }}} .
$$

Let us discuss first QGSJET. In this model cross sections are calculated on the basis of the quasieikonal approximation [6, 15-17], and it is found that

$$
\begin{aligned}
\sigma_{\text {tot }} & \propto \ln ^{2}(s), \\
\sigma_{\text {inel }} & \propto \ln ^{2}(s), \\
\sigma_{\text {diff }} & \propto \ln ^{2}(s),
\end{aligned}
$$

where $s$ is the (square of the) center of mass energy. Then, from equation (3) it follows that in the QGSJET case one has $F_{\text {diff }} \approx$ const. when $s \rightarrow \infty$, in accordance with the corresponding curve in figure 3 .

On the other hand, for the SIBYLL case $[1,5,18,19]$ and in the high energy limit, the total and inelastic cross sections behave again proportional to $\ln ^{2}(s)$ as in equations (4) and (5), but the diffractive cross section grows logarithmically, that is

$$
\sigma_{\text {diff }} \propto \ln (s)
$$

Then, from equations (3), (4), and (7), it is straightforward to see that $F_{\text {diff }} \rightarrow 0$ when $s \rightarrow \infty$, similarly as in the SIBYLL curve displayed in figure 3 .

It should also be noticed that the experimental diffractive cross section presents a strong saturation effect starting around $\sqrt{s}=50 \mathrm{GeV}$ (see for example figure 1 of reference [20]). This effect is probably related to unitarization. The saturation in diffraction is in contrast with the growing in energy of the total cross section [20]. Even without entering a more detailed theoretical discussion, one can expect that the diffractive component of the cross 

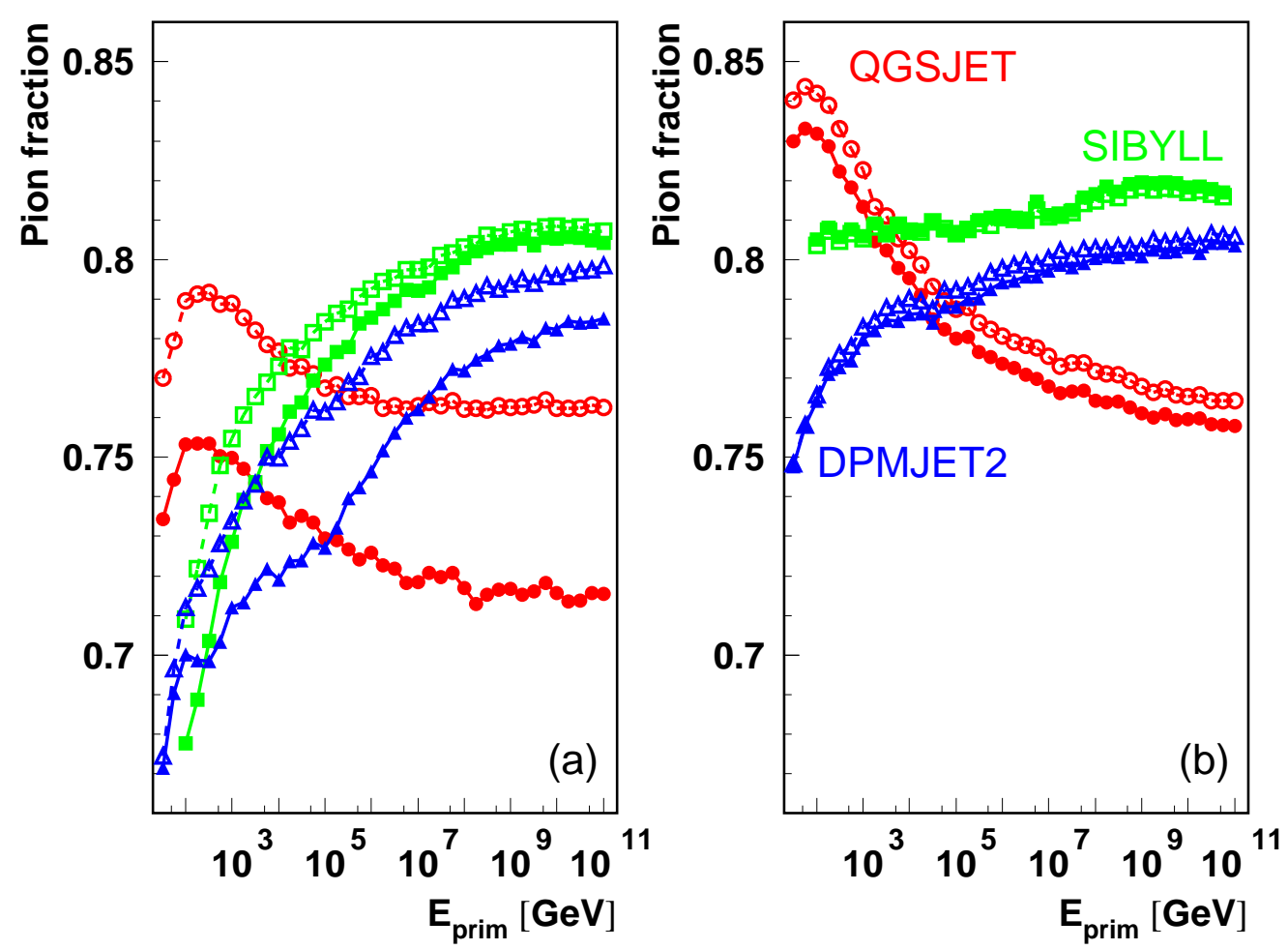

Figure 4: Average fraction of pions produced in hadronic collisions versus primary energy, in the cases of proton-air (a) and pion-air (b) collisions. The solid (open) symbols correspond to averages over all (non-diffractive) events.

section looses protagonism with energy, leading in turn to a relative fraction of diffractive events that decreases with primary energy, corresponding qualitatively to the SIBYLL or DPMJET cases plotted in figure 3 .

The hadronic models studied here present noticeable differences when considering the composition of the secondaries generated in nuclear collisions. A useful quantitative measure of the kind of particles emerging from such collisions is the fraction of pions, that is, the total number of pions (charged and neutral) divided by the total number of secondaries.

In figure 4 the fractions of pions for proton-air (a) and pion-air (b) collisions are plotted as a function of the primary energy. The differences between models are evident: The SIBYLL and DPMJET fractions rise with energy, while the corresponding one for QGSJET decreases after reaching a maximum at relatively low $E_{P}$. Additionally, the largest differences correspond to energies around and below $1 \mathrm{TeV}$, a region of particular importance in the case of air showers due to the existing direct correlation between the low energy pion production 
and the number of muons in the shower. It should be noticed that the discrepancies in the pion fractions coming from different models are also present at low energies, as reported in reference [12].

\section{B. Energy and transverse momentum}

In a normal hadronic collision producing many secondaries, the energies of the emerging particles distribute broadly within the range of all possible energies $E \leq E_{P}$. This fact is

illustrated in figure 5, containing the energy distributions of pions and nucleons, in the very representative case of $100 \mathrm{GeV}$ proton-air collisions.

The pion energy distributions are unimodal, and are centered at energies around 2 to $5 \mathrm{GeV}$. Notice the larger area in the QGSJET case, indicating that the average number of pions produced by this model is larger than the corresponding ones for SIBYLL and DPMJET.

The distributions for nucleons present a more complicated structure, product of the more involved mechanisms of nucleon production that enter in action in the different models. These distributions are made up of two components clearly distinguishable: (i) A neat peak at $E_{\mathrm{sec}} \simeq E_{P}$, that corresponds to leading nucleons emerging from diffractive events. (ii) $\mathrm{A}$ widely spread distribution that corresponds to inelastic production of nucleons and antinucleons. This part of the distribution is in general multimodal, indicating the coexistence of several production mechanisms with different average secondary energy.

A detailed theoretical explanation of the characteristics of these distributions is beyond the purpose of the present work. The interested reader can find further details in the references $[3,4,6,7]$.

For meson primaries, the energy distribution of secondary nucleons (not plotted here for brevity) acquires a simpler structure (much like the pion energy distribution) with virtually no secondaries having $E_{\mathrm{sec}} \simeq E_{P}$. This is a clear consequence of the fact that in this case the diffractive interactions involve mesons as leading particles.

For larger primary energies, the secondary energy distributions (not plotted here) maintain approximately most of the features of the already described distributions at $100 \mathrm{GeV}$, but extending always in the entire allowable energy range. In the case of the energy distribution of pions, the central value increases continuously with the primary energy. 

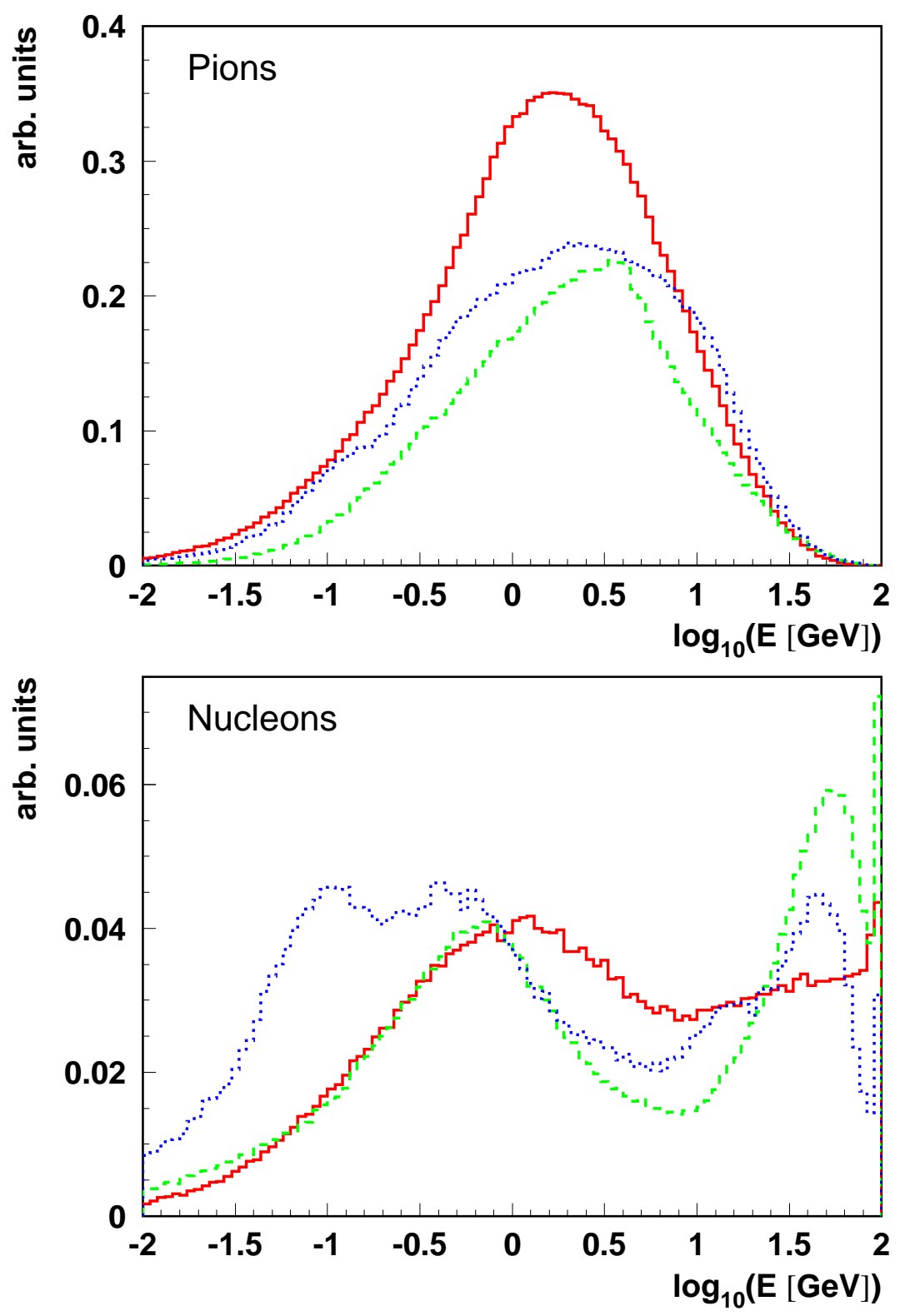

Figure 5: Energy distributions of secondary pions and nucleons in the case of $100 \mathrm{GeV}$ protonair collisions. The solid, dashed, and dotted histograms correspond to QGSJET, SIBYLL, and DPMJET2, respectively.

Another quantity of interest to our analysis is the distribution of the fraction of energy carried by the leading secondary, $f_{L}$ (see the definition at section II).

Figure 6 displays typical $f_{L}$ distributions. The plots include distributions for QGSJET, SIBYLL, and DPMJET for proton projectiles at several representative energies.

The sharp peaks around $f_{L}=1$ are the distinctive signature of the diffractive events (The 


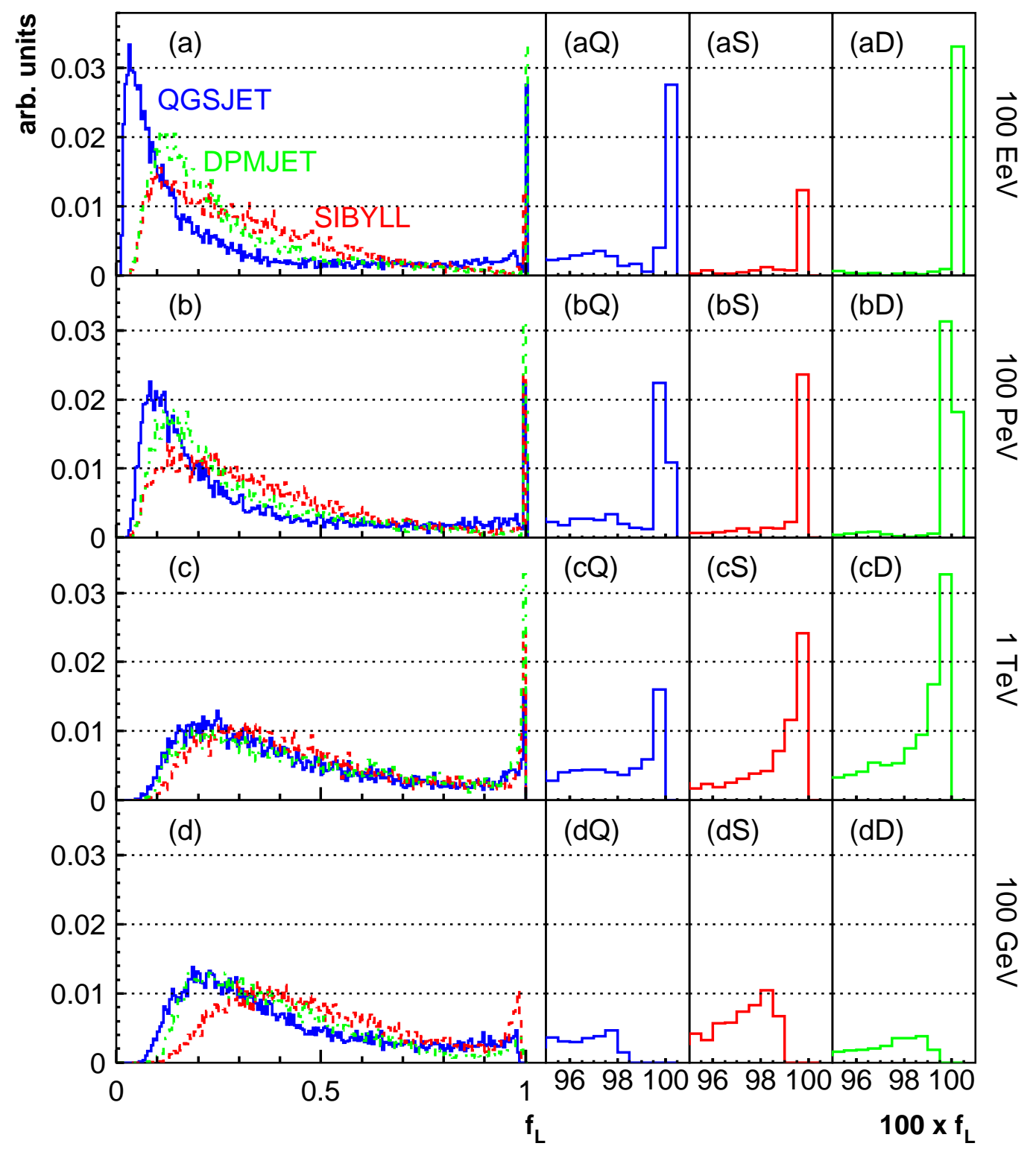

Figure 6: Leading energy fraction distributions for proton-air collisions at different representative primary energies.

small plots at the right part of figure 6 show these peaks in detail. Notice that the areas of such peaks are correlated to the respective diffractive event factions plotted in figure 3 . On the other hand, in the properly inelastic collisions the available energy is shared among many secondaries, leading to a fluctuating $f_{L}$ that distributes in the whole $[0,1]$ range.

It is worth noticing the particular shape of the QGSJET distribution at the highest energies (figure 6 (a)), that presents two noticeable peaks located at both $f_{L} \sim 0$ and 

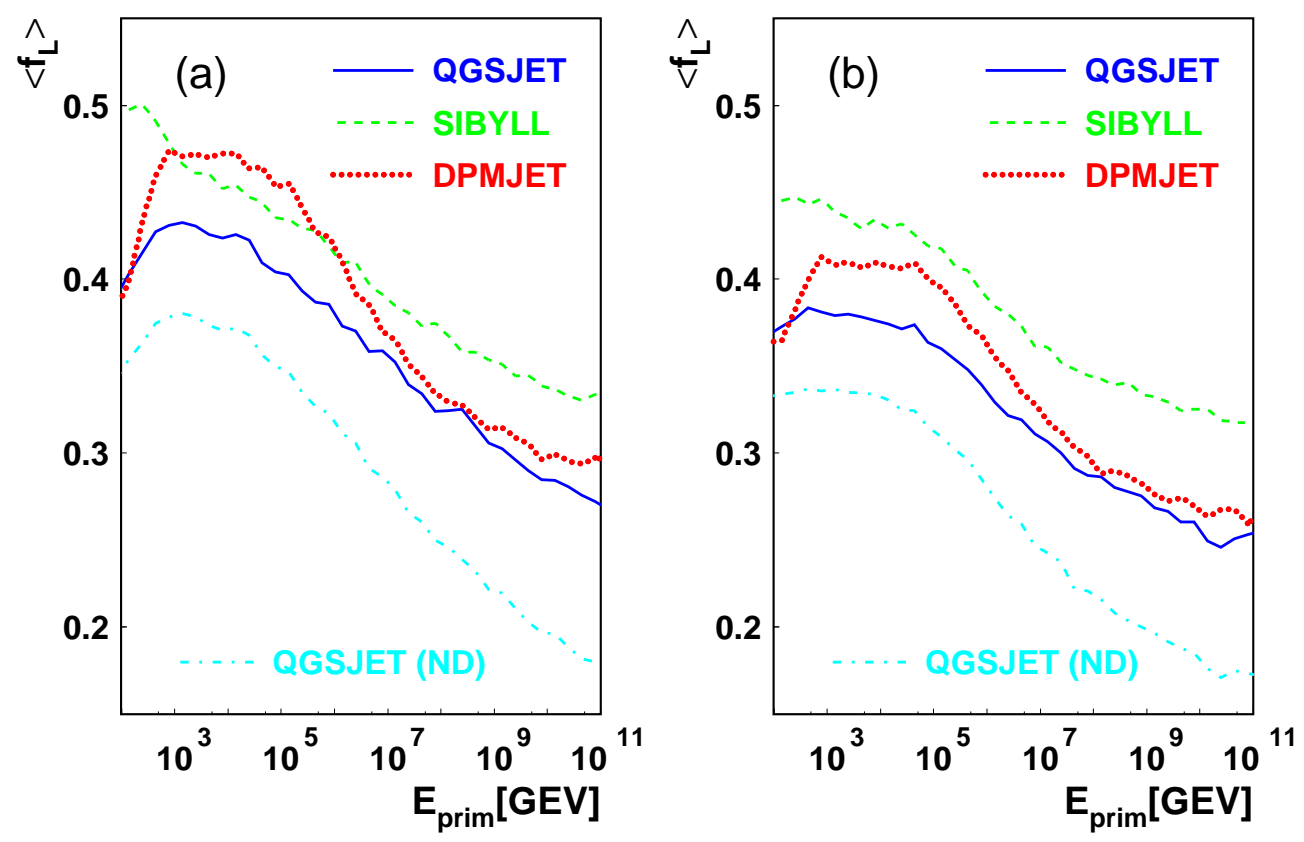

Figure 7: $\left\langle f_{L}\right\rangle$ versus $E_{\text {prim }}$ for proton-air (a), and pion-air (b) collisions

$f_{L} \sim 1$ extremes. The concentration of events around $f_{L} \sim 0$ is clearly more accentuated than in the cases of SIBYLL or DPMJET. This difference is directly correlated with the very large number of secondaries produced by QGSJET in inelastic collisions at the highest energies.

The plots in figure 7 also illustrate this particular characteristic of QGSJET. In this figure the mean $\left\langle f_{L}\right\rangle$ is plotted as a function of the primary energy for the cases of proton and pion projectiles. The graphs include two curves for QGSJET, namely, the general average, and the average excluding diffractive processes. This last one indicates clearly that the fraction of energy carried away by the leading particle is sensibly lower than in every other case, in agreement with the data displayed in figure 6 for proton collisions at representative fixed energies.

Notice also that at the highest energies the largest $\left\langle f_{L}\right\rangle$ corresponds to SIBYLL, despite its very low diffractive probability (see figure 3 ).

We conclude our study of individual collisions by analyzing the transverse momenta of the secondary particles.

The transverse momentum distributions, conveniently described by means of pseudorapidity distributions, are significantly correlated with shower observables like the lateral 

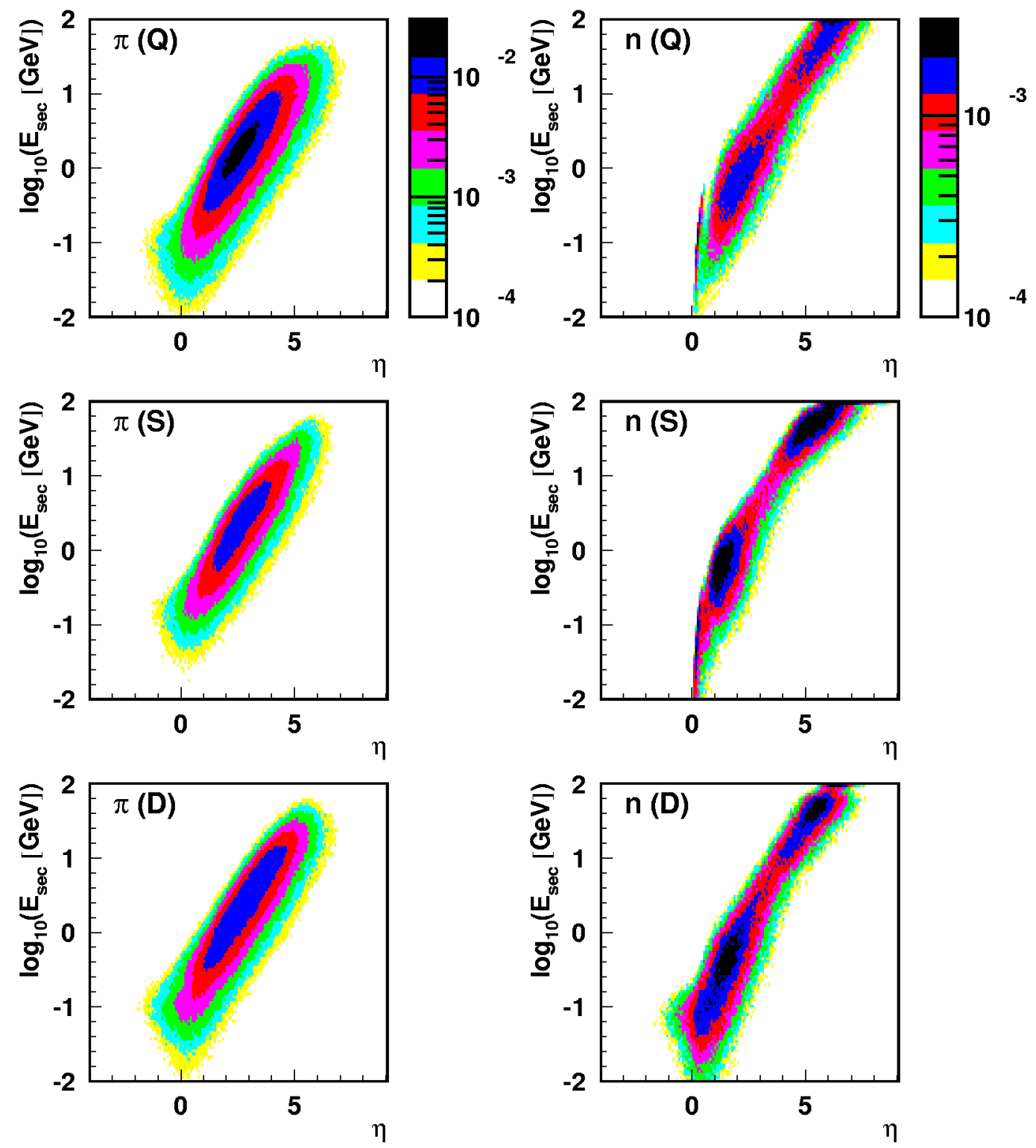

Figure 8: Secondary pseudorapidity versus energy plots for the case of $100 \mathrm{GeV}$ proton-air collisions. The plots in the left (right) column correspond to pion (nucleon) distributions, for collisions generated with QGSJET (Q), SIBYLL (S), and DPMJET (D). The scales are the same at each column.

distribution of muons at large distances from the core [21], and constitute therefore an additional source of uncertainty to be taken into account when estimating systematic errors associated to Monte Carlo estimations of shower observables.

Figures 8 and 9 contain $\eta \times E_{\text {sec }}$ two-dimensional distributions for proton-air collisions at 

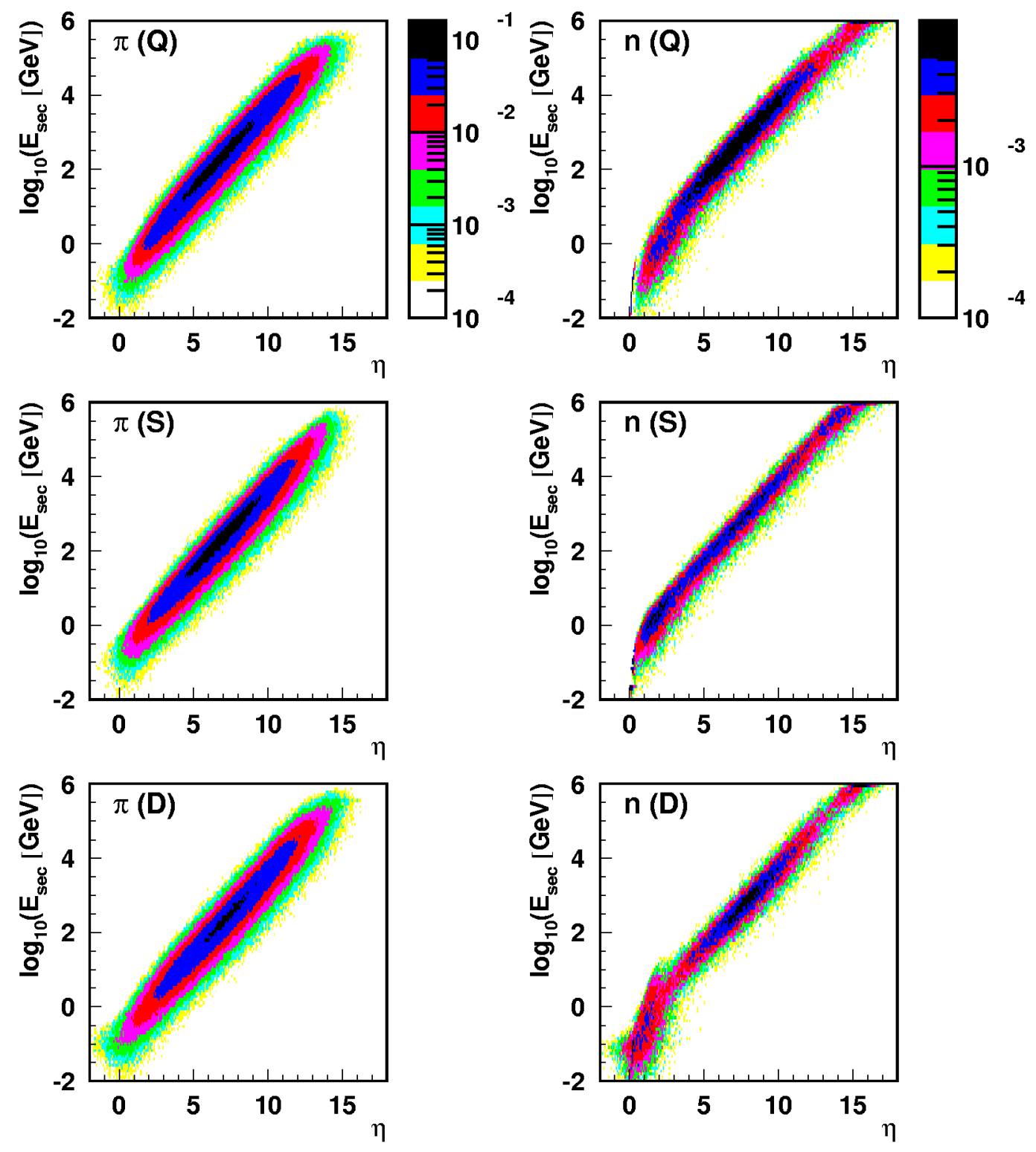

Figure 9: Same as figure 8 but for $1 \mathrm{PeV}$ proton-air collisions.

$100 \mathrm{GeV}$ and $1 \mathrm{PeV}$ respectively.

The simplest distributions are the ones corresponding to secondary pions. At each secondary energy the pseudorapidity $\eta$ distributes around a central value approximately like a Gaussian, but presenting however a longer tail towards the region of large $\eta$. The mean pseudorapidity increases with $\log E_{\mathrm{sec}}$ approximately in a linear form.

On the other hand, the distributions for nucleons are more complex, and there are evident differences between the studied hadronic models. In particular, both SIBYLL and QGSJET 

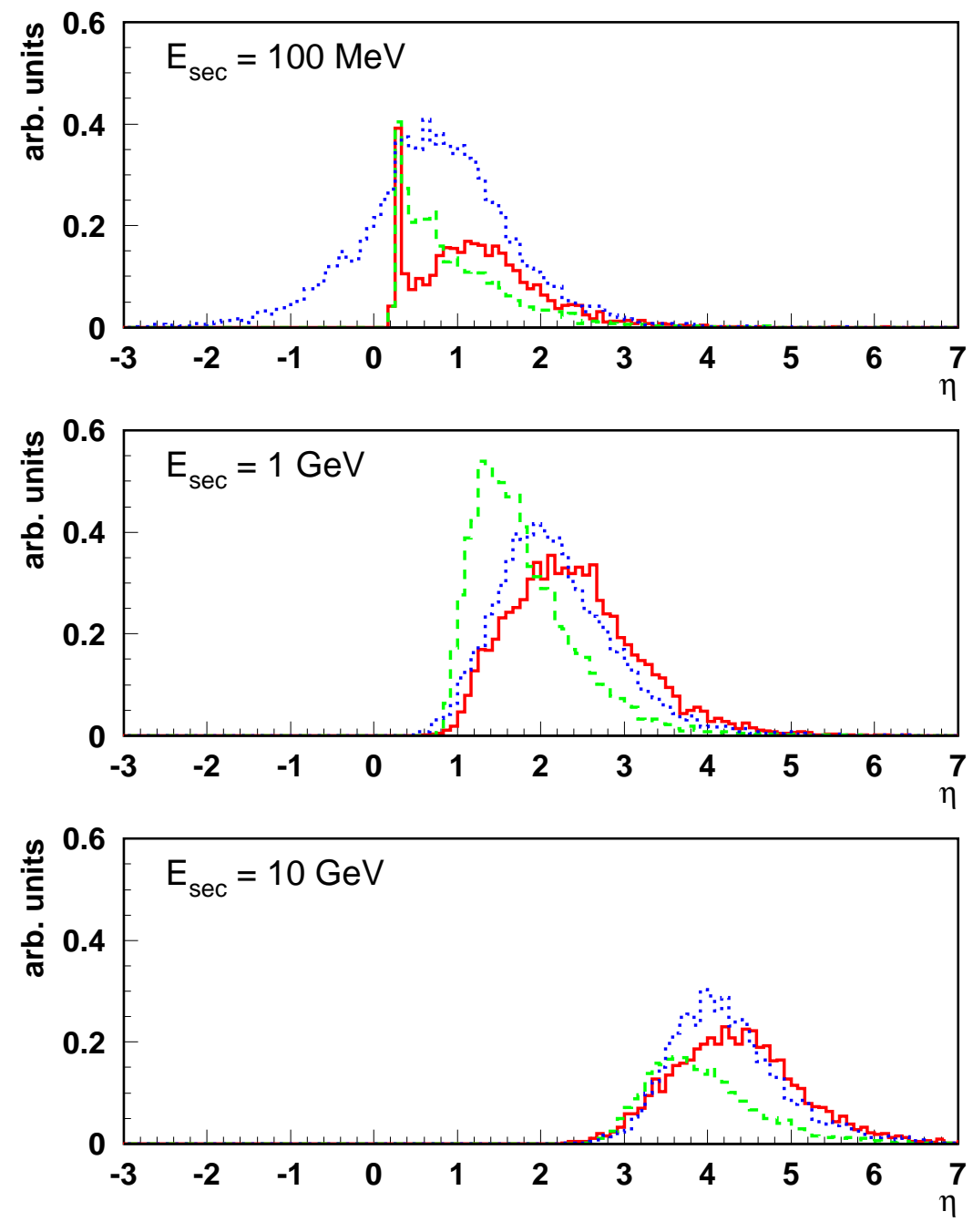

Figure 10: Some cuts around representative secondary energies of the $\eta \times E_{\mathrm{sec}}$ distributions of figure 8. The full, dashed, and dotted lines correspond to the QGSJET, SIBYLL, and DPMJET cases, respectively.

distributions do not produce recoiling nucleons, that is, there are no $\eta<0$ events, as it shows up clearly in the figures. This is not the expected behavior of the secondaries which can eventually emerge as recoiling particles, especially for those having low energies. Notice that, on the other side, DPMJET is capable of generating such recoiling particles

In figure 10 we show three representative $\eta$ distributions corresponding to three slices of the distributions of the right column of figure 8, at $100 \mathrm{MeV}, 1 \mathrm{GeV}$, and $10 \mathrm{GeV}$, respectively. 
In the $100 \mathrm{MeV}$ distributions the lack of recoiling nucleons in the SIBYLL and QGSJET cases is most evident. Notice also that in the remaining case the distributions are similar but not completely coincident.

\section{EFFECT ON SHOWERS OBSERVABLES}

The second step in our analysis is to study the impact that different alternatives for modeling the diffractive hadronic interactions have on common air shower observables.

We have used the AIRES program [22] to simulate proton and iron induced showers with different primary energies, and using QGSJET01 and SIBYLL 2.1 to process the high energy hadronic interactions [24].

In the previous section we have shown that the fraction of diffractive events was one of the most outstanding differences between the tested hadronic codes (see for example figure 3). We consider therefore that it is worthwhile to obtain quantitative estimations of the impact of the diffractive interactions on shower observables. To this end, we have run simulations using SIBYLL or QGSJET to process the hadronic interactions, with two different configurations: (i) normal setting mixing diffractive and non-diffractive events. (ii) disabling diffractive interactions.

\section{A. Longitudinal development}

Because of their active role in energy transport, the diffractive interactions have a direct impact on the global shower development. This fact shows up clearly in figures 11 and 12 where the number of charged particles is plotted versus the atmospheric depth, in the cases of $10^{17}$ and $10^{20} \mathrm{eV}$ vertical proton showers, respectively. The plots were done using data coming from simulations performed with AIRES linked to QGSJET (a) and SIBYLL (b). As expected, when the diffractive interactions are disabled (dotted lines), the showers develop earlier than in the normal case. This implies a displacement in the position of the maximum, $X_{\max }$, that amounts approximately to $20 \mathrm{~g} / \mathrm{cm}^{2}$ for iron and $30 \mathrm{~g} / \mathrm{cm}^{2}$ for proton $\left(10 \mathrm{~g} / \mathrm{cm}^{2}\right.$ for both iron and proton) for QGSJET (SIBYLL) simulations.

The shift in the position of the shower maximum, due to the suppression of diffractive interactions is significant at all primary energies. This is illustrated in figure 13 where $X_{\max }$ 

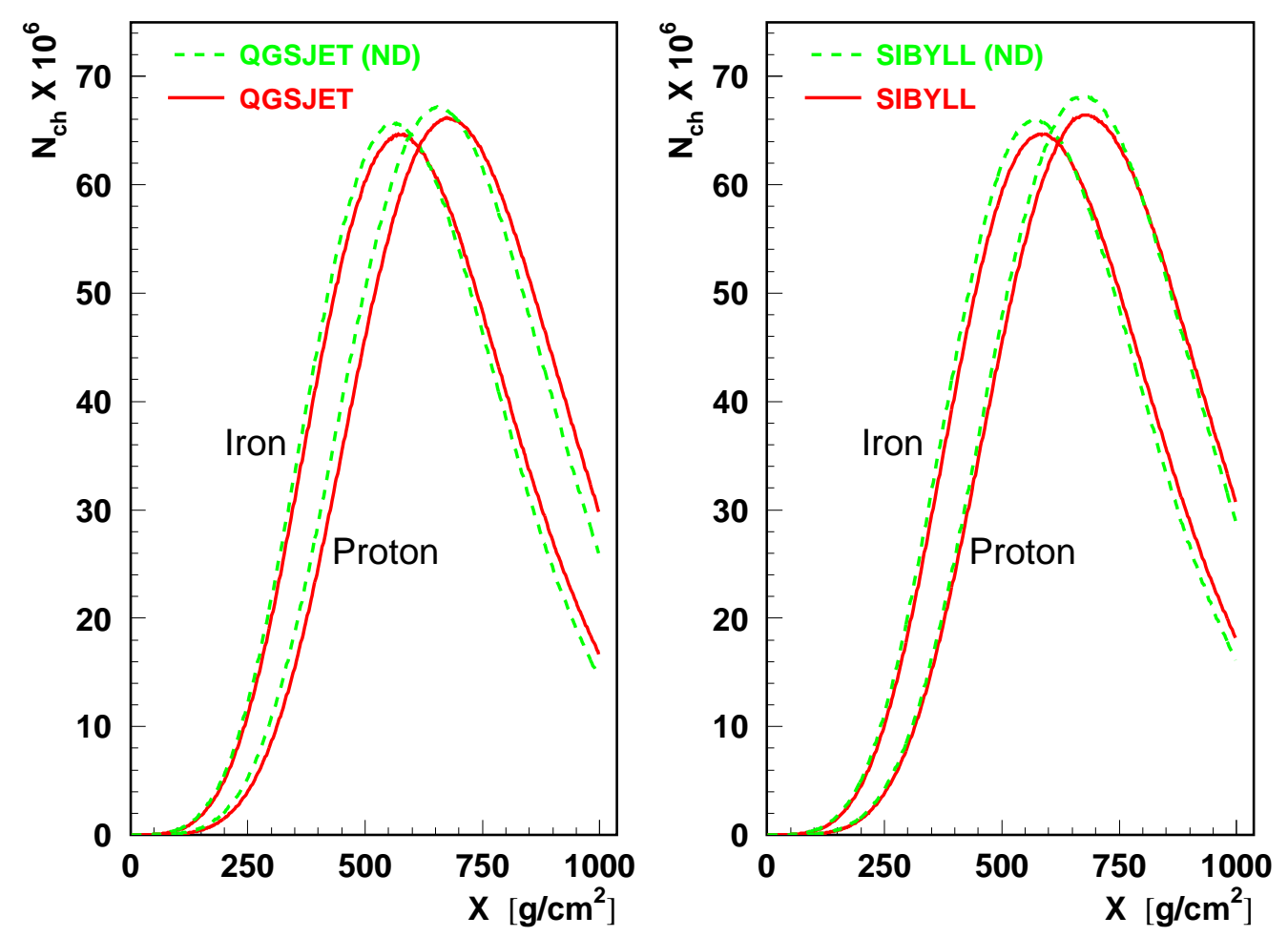

Figure 11: Average longitudinal development of $10^{17} \mathrm{eV}$ proton and iron showers. The simulations were performed using AIRES linked to QGSJET01 (a), or SIBYLL 2.1 (b).

is plotted versus the primary energy. The lines represent simulations of proton and iron showers enabling (solid lines) or disabling (dashed lines) the diffractive interactions. We have also plotted some available experimental data for reference [2].

We can clearly see that in the entire covered range of primary energies the suppression of diffractive interactions always produce a non negligible reduction of $X_{\max }$. It is clear that the fully non-diffractive simulations are unrealistic, but they are useful to quantitatively estimate a rough upper bound of the uncertainty of $X_{\max }$ that can be expected due to the uncertainties associated with diffractive hadron-nucleus interactions, especially at the highest energies.

Notice also that the differences for SIBYLL are generally smaller than the corresponding ones for the QGSJET case. This is correlated with the fact that in SIBYLL the diffractive interactions have a very small probability, in comparison with QGSJET, as discussed in section III A.

The diffractive interactions have also a direct impact on the development of the hadronic 

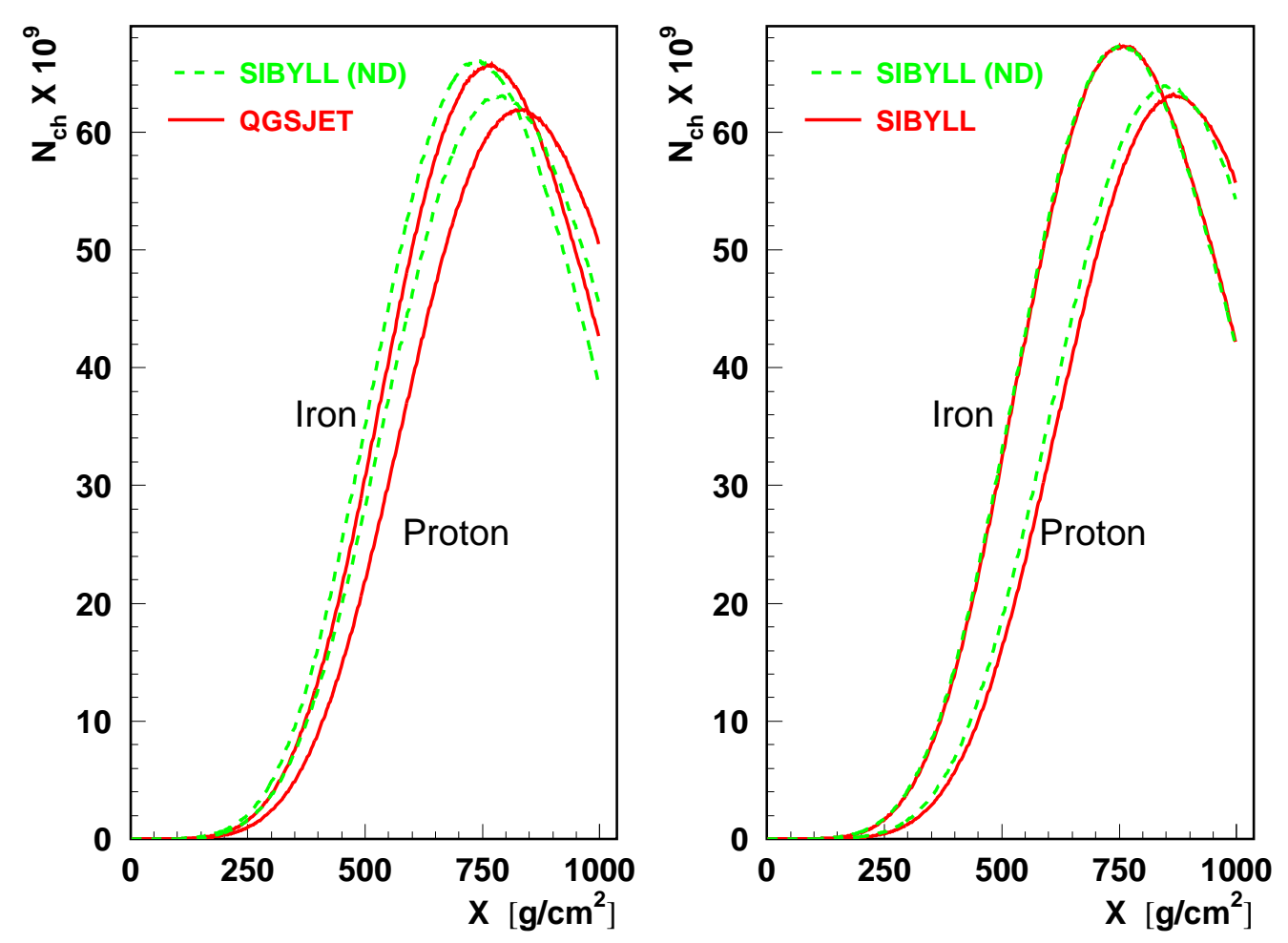

Figure 12: Same as figure 11 but for $10^{20} \mathrm{eV}$ showers.

and muonic components of the showers. This fact is illustrated in figure 14 where the numbers of pions and muons are plotted as functions of the atmospheric depth, in the case of showers initiated by $10^{20} \mathrm{eV}$ protons. For both SIBYLL and QGSJET cases, the number of pions increases when the diffractive interactions are disabled (figures 14a and 14b). This can be clearly understood because the bulk of the pions are produced at inelastic hadronic collisions, whose number is enlarged when diffraction is switched off.

Muons come mainly from decays of charged pions and therefore it can be expected that a larger number of pions leads to an increase in the number of muons. This feature is clearly illustrated at figures $14 \mathrm{c}$ and $14 \mathrm{~d}$, that show that the muon numbers for the non diffractive case are larger than the respective ones for the normal simulations.

\section{B. Lateral distributions}

The lateral distribution of particles reaching ground is a key observable whose accurate determination is essential for the calibration of surface array detectors $[9,12,13]$, like the 

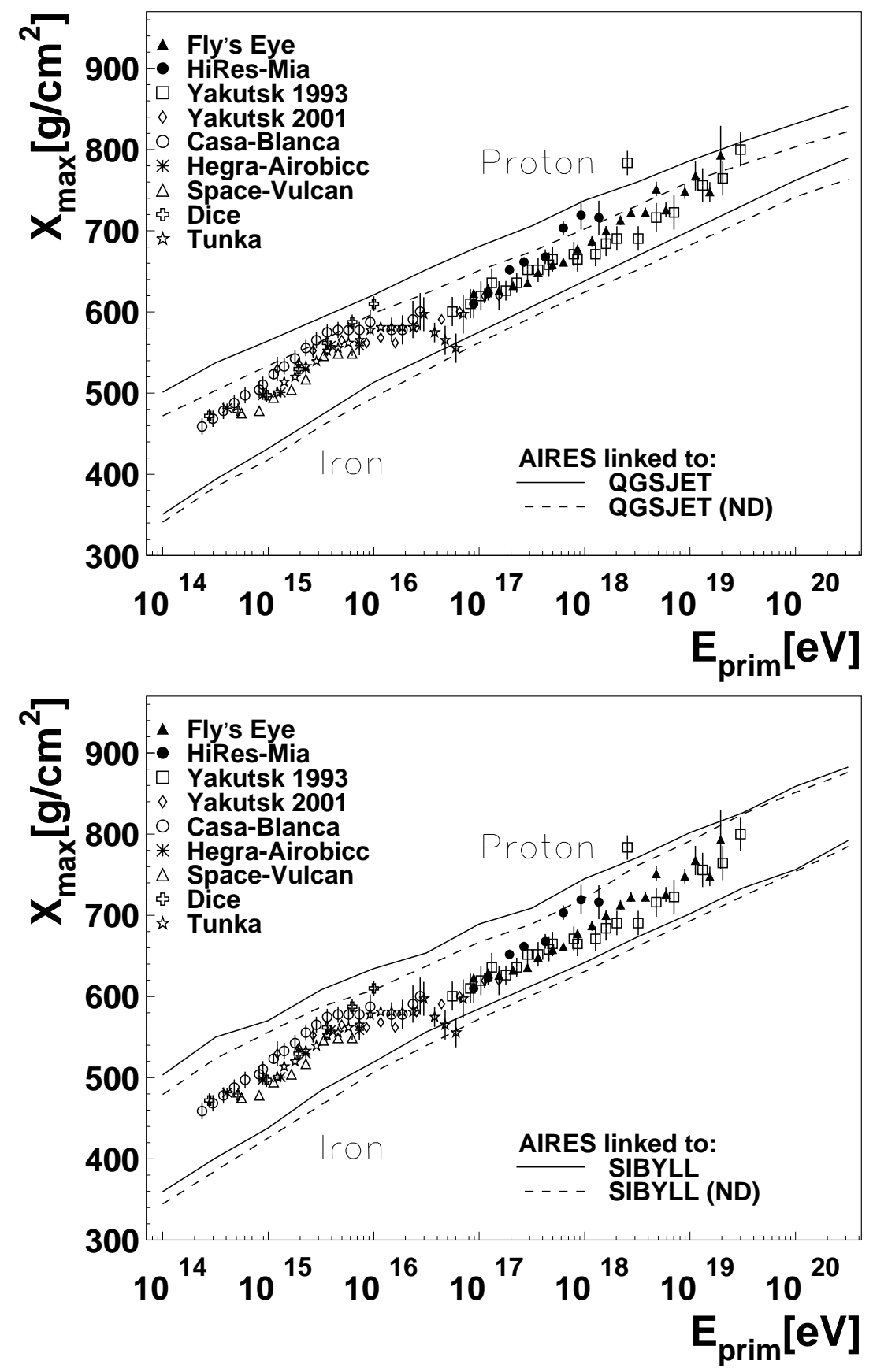

Figure 13: Average shower maximum versus primary energy.

water Cerenkov tanks of the Auger experiment [23] for example. In such detectors the primary energy is estimated from the signal measured at a determined distance from the shower core, 1000 meters for example. In the particular but important case of water Cerrenkov detectors, the detected signal comes mainly from the electromagnetic particles (gammas, electrons and positrons), and the muons. In a simulation, the muonic part depends strongly 

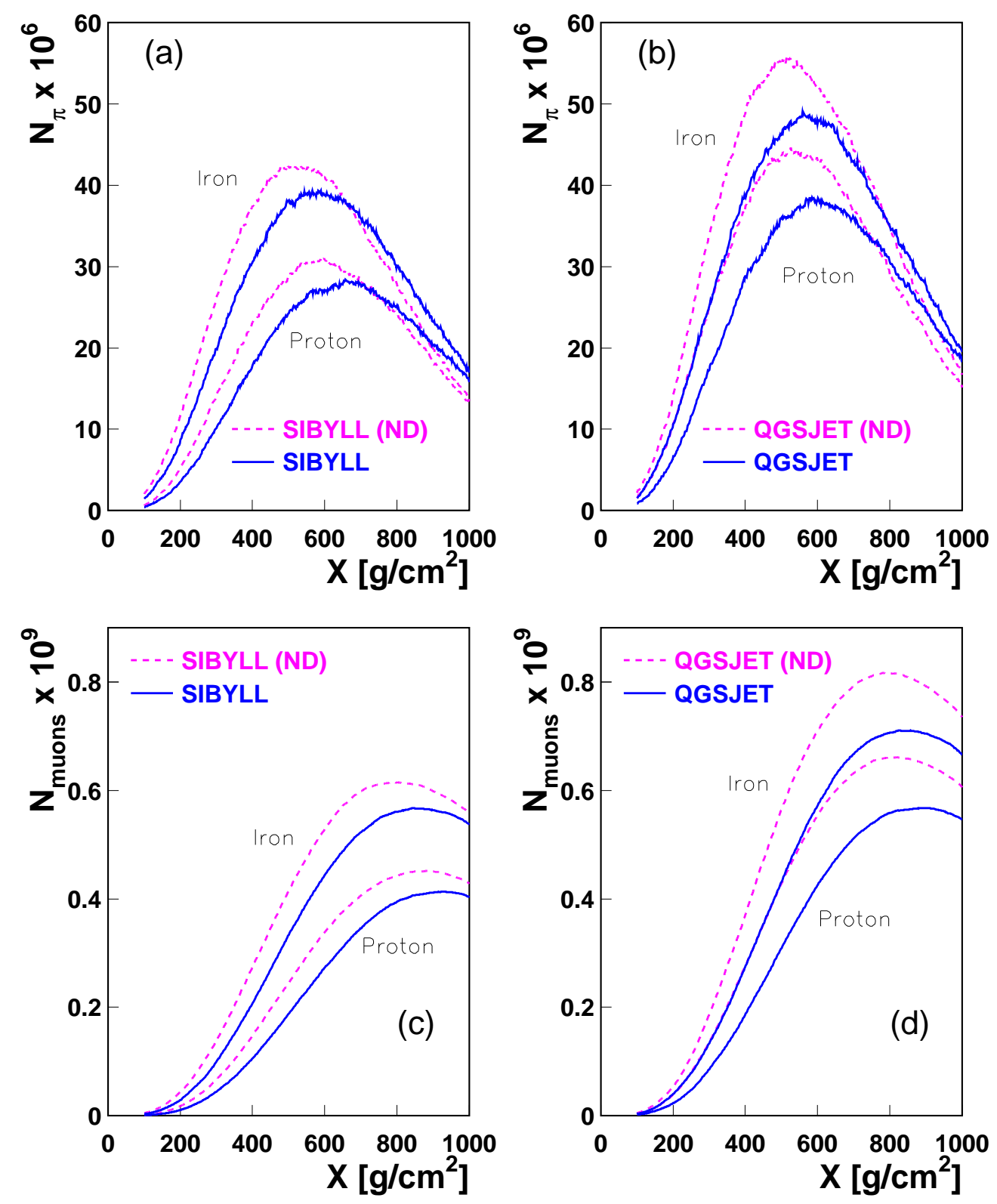

Figure 14: Longitudinal development of charged pions and muons for showers initiated by $10^{20} \mathrm{eV}$ protons.

on the characteristics of the hadronic model used. This is due to the fact that the dominant channel for muon production is pion decay, so the number of produced muons is directly correlated with the number of charged pions which in turn appear mainly during hadronic collisions.

For these reasons we have included in our study an analysis of the correlations between the 

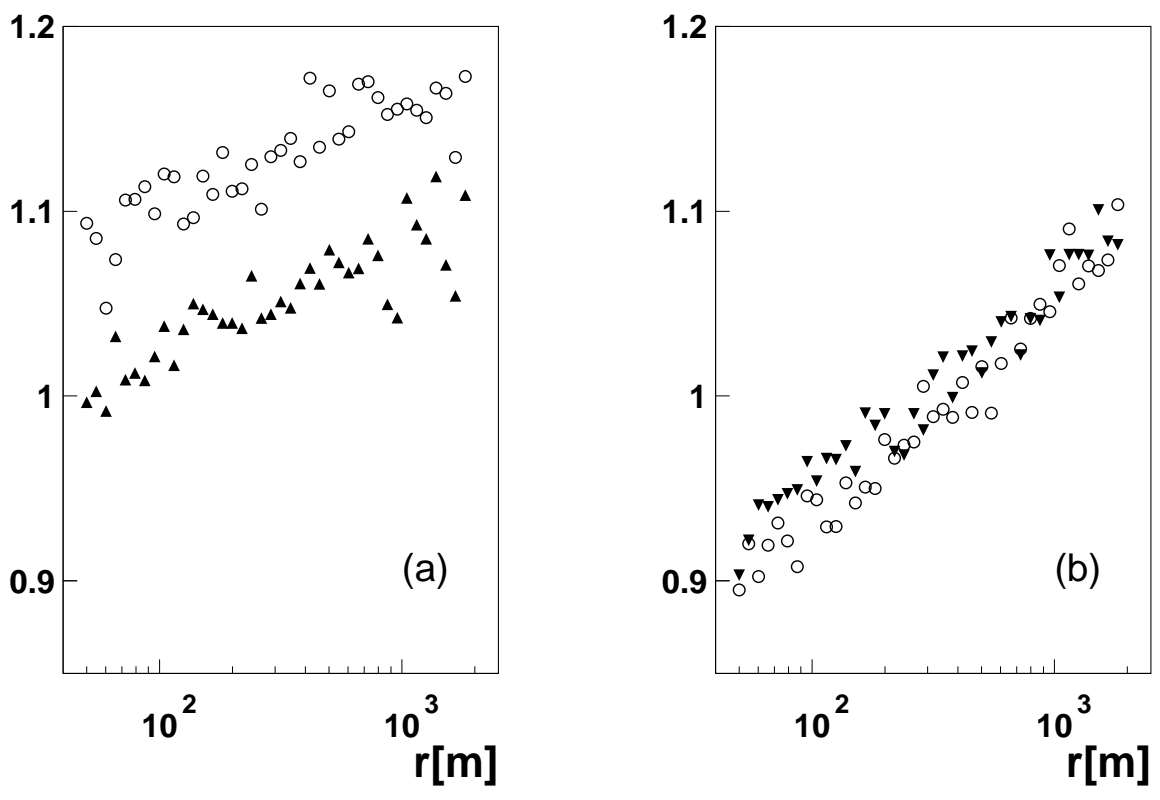

Figure 15: Ratio between muon densities simulated disabling and enabling diffractive interactions, plotted as a function of the distance to the shower core. The triangles (circles) correspond to $10^{17}$ eV iron (proton) primaries. The simulations were performed using AIRES linked to QGSJET (a), or SIBYLL (b), and correspond to vertical showers observed at a ground altitude of $1000 \mathrm{~g} / \mathrm{cm}^{2}$.

lateral distribution of muons and the diffractive interactions that take place during shower development.

Let $\rho_{\mu}(r)$ be the density of muons at a given distance $r$ from the shower core. In figure 15 we have plotted the ratio $\rho_{\mu}^{(\mathrm{ND})}(r) / \rho_{\mu}^{(\mathrm{D})}(r)$ between muon densities simulated disabling and enabling diffractive interactions, as a function of $r$. When this ratio is 1 , this means that enabling or disabling the diffractive interactions that could take place during the development of the showers, does not alter the muon density at ground.

We can see, however, that our simulations indicate that this ratio is, in general, different from 1, and that its behavior depends on the hadronic model used.

In the QGSJET case (figure 15a), the ratio increases with $r$, and depends on the nature of the primary. At $r=1000 \mathrm{~m}$ the ratio is approximately 1.05 (1.15) for proton (iron) primaries.

Figure 15b illustrates the SIBYLL case, characterized by a ratio that increases with $r$. It is smaller that 1 when $r<300 \mathrm{~m}$, and greater than one otherwise. No significant composition 
dependencies are present in this case, that gives 1.07 for both proton and iron primaries at $r=1000 m$.

From both plots one can conclude that the uncertainty in the diffractive cross sections imply an uncertainty of about $10 \%$ in the muon density at $1000 \mathrm{~m}$ from the core. Additionally, the slope of $\rho_{\mu}(r)$ is found to be significantly dependent on the diffractive cross section. This fact should be taken into account when considering the accuracy of the primary mass estimation algorithms that are based on measurements of the shape of the lateral distributions.

It is also important to mention that the preceding precentages depend on various shower parameters like primary energy, inclination, and ground altitude. Therefore those figures should be considered only as qualitative indicators.

As mentioned before, the electromagnetic component of the particles reaching ground is affected in a lesser degree when switching on and off the diffractive interactions. While a complete analysis of the variations of measurable signals at ground detectors is beyond the scope of this work, we can nevertheless mention that a simple analysis leads to the conclusion that the relative variations of the total signal are qualitatively similar to the muon densities plotted in figure 15, with an uncertainty of about $10 \%$ at $1000 \mathrm{~m}$ from the core, in the representative case of $10^{20} \mathrm{eV}$ proton showers inclined $30 \mathrm{deg}$.

\section{CONCLUSIONS}

We have performed an extensive analysis of some of the characteristics of commonly used hadronic collision simulation packages, namely SIBYLL, QGSJET, and DPMJET.

The contribution of diffractive processes, as defined in section II, was studied with particular detail, including an analysis of their impact on several shower observables.

The data obtained from series of hadron-nucleus collisions simulated using the mentioned models with identical initial conditions indicate that there are significant differences between models for observables such as mean multiplicity, inelasticity, fraction of pions, energy distribution of secondaries, as well as pseudorapidity distributions in some cases.

Such differences exist for all the primary energies that have been studied, which include the range of moderate energies where experimental data do exist. At such energies (of the

order of $100 \mathrm{GeV}$ ) the most noticeable differences correspond to the mean multiplicity (see 
inset of figure 2, and fractions of pions (see figure 4).

The average fractions of diffractive events detected at given primary energies have also been studied. Our analysis puts in evidence enormous differences between models (see figure 3): for QGSJET the fraction of diffractive events rises slowly with energy, passing from about $10 \%$ at $E_{\text {prim }}=100 \mathrm{GeV}$ to $13 \%$ at $E_{\text {prim }}=10^{20} \mathrm{eV}$. On the other hand, for SIBYLL this fraction diminishes with energy, from about $12 \%$ at $E_{\text {prim }}=200 \mathrm{GeV}$, down to about $1 \%$ at $E_{\text {prim }}=10^{20} \mathrm{eV}$. DPMJET presents an "intermediate" behavior as illustrated in figure 3.

The fraction of diffractive events is directly related to the diffractive to total cross section ratio. The data plotted in figure 3 seem to indicate that the different models have significantly different ways of extrapolating those cross sections for the case of extremely large energies, while presenting qualitatively similar values at primary energies around $1 \mathrm{TeV}$.

Our study is completed with an analysis of the impact of the diffractive events on common shower observables. We have run several shower simulations using SIBYLL and QGSJET, enabling or disabling the diffractive events, with the main purpose of extracting conclusions about the maximum impact that the uncertainty in the diffractive probability can have on the considered observables.

We have found a moderate but not negligible impact for both the position of the shower maximum, $X_{\max }$, and the lateral distribution of muons, $\rho_{\mu}(r)$. In this last case, it is remarkable the change of slope detected when changing the probability of diffractive events.

It is worthwhile mentioning that in the case of $X_{\max }$, the detected differences are of the order of about $2 \%$, and this figure is of the same order of magnitude than other uncertainties in $X_{\max }$ connected with the hadronic model, that have been already reported in a previous work [1].

The detected discrepancies between models call for further studies, both theoretical and experimental. In this last case, the possibility of measuring fractions of diffractive events and multiplicities at primary energies larger than $1 \mathrm{TeV}$ will certainly help to improve the constraints needed to validate a given model.

\section{Acknowledgments}

We are indebted to T. Gaisser, R. Engel, H. Fanchiotti and R. Sassot for useful discussions and comments. This work was partially supported by ANPCyT and Fundación Antorchas 
of Argentina, and SUPERA-SEP and CONACYT of Mexico.

[1] J. Knapp, D. Heck, S. J. Sciutto, M. T. Dova, M. Risse. Astrop. Phys., 19, 77 (2003).

[2] R. Engel, Nucl. Phys. B (Proc. Suppl.), 122, 40 (2003).

[3] R. Engel, T. K. Gaisser, T. Stanev, Proc. 26th ICRC (Utah), 1, 415 (1999) (AIP Conference Proceedings, New York (1999)).

[4] J. Engel, T.K. Gaisser, T. Stanev and Paolo Lipari, Phys. Rev. D, 46, 5013 (1992).

[5] R. Fletcher, T. K. Gaisser, Paolo Lipari, and Todor Stanev, Phys. Rev. D, 50, 5710 (1994).

[6] N. N. Kalmykov, S. S. Ostapchenko, A. I. Pavlov, Nucl. Phys. B (Proc. Suppl.), 52B, 17 (1997).

[7] D. Heck, Proc. 27th ICRC (Hamburg), 1, 433 (2001) (Copernicus Gesellschaft, Hamburg, 2001).

[8] J. Ranft. New features in DPMJET II.5, Siegen preprint (1999).

[9] J. Ranft. Phys. Rev. D, 51, 64 (1995).

[10] S. Roesler. R. Engel and J. Ranft: (hep-ph/0012252), in Proc. of Conf. on Advanced Montecarlo Physics Particle Transport Simulations and Applications (MC 2000), Lisbon, Portugal, 23-26 Oct. 2000.

[11] S. Roesler. R. Engel and J. Ranft: Proc. of 27th, ICRC (Hamburg), 446 (2001) (Copernicus Gesellschaft, Hamburg, 2001).

[12] S. J. Sciutto, in Proc. of the X Mexican School of Particles and Fields, AIP Conference Proceedings vol. 670, New York (2003).

[13] H. J. Drescher, M. Hladik, S. Ostapchenko, T. Pierog and K. Werner, Phys. Rep., 350, 93 (2001).

[14] L. A. Anchordoqui, M. T. Dova, L. N. Epele, S. J. Sciutto. Phys. Rev. D, 59, 094003 (1999).

[15] A. B. Kaldakov and K. A. Ter-Martirosyan, Sov. J. Nucl. Phys., 40(1), 135 (1984).

[16] A. B. Kaldakov and O. I. Piskunova, Z. Phys., C30, 145 (1988).

[17] V. Zoller, Sov. J. Nucl. Phys., 48, 361 (1988).

[18] T. K. Gaisser and T. Stanev, Phys. Lett., B219, 375 (1989).

[19] R. S.Fletcher, Phys. Rev. D, 46, 187 (1992).

[20] K. Goulianos, hep-ph/0407035. Presented at Les Rencontres de Physique de la Valle d'Aoste, 
February 2004.

[21] S. J. Sciutto, Talk presented at the Pierre Auger Collaboration meeting, Malargüe, Argentina, October 2001.

[22] S. J. Sciutto, Proc. 27th ICRC (Hamburg), 1, 237 (2001) (Copernicus Gesellschaft, Hamburg, 2001); see also www.fisica.unlp.edu.ar/auger/aires .

[23] The Pierre Auger Observatory Collaboration, Nucl. Instr. and Meth. A, 523, 50 (2004).

[24] AIRES uses a built-in algorithm to process the low energy $(E<100 \mathrm{GeV})$ hadronic and photonuclear interactions. High energy photonuclear processes are simulated via calls to the corresponding external hadronic package. A detailed description of the organization and features of AIRES is provided in reference [22]. 